\title{
Investigations of a reproductive processing advantage in memory
}

\author{
Cory J. Derringer ${ }^{1} \cdot$ John E. Scofield ${ }^{2} \cdot$ Bogdan Kostic $^{2}$
}

Published online: 1 May 2017

(C) Psychonomic Society, Inc. 2017

\begin{abstract}
Previous work has shown that processing words for their survival value improves memory. If this survival processing effect reflects an evolutionary adaptation in memory, processing words for their reproductive value should also improve memory. Across three experiments, participants rated words for their relevance in reproductive scenarios. In Experiment 1, participants rated adjectives (traits) for their relevance in finding a mate, evaluating a coworker, or in terms of their pleasantness. Mate processing produced better memory than pleasantness ratings, but not coworker processing. In Experiment 2, participants rated traits for their relevance in detecting sexual or emotional infidelity. Neither processing condition produced better memory compared to pleasantness ratings, but there were several unpredicted interactions involving participant sex and jealousy responses. In Experiment 3, participants rated gifts for their appropriateness in a romantic date or a housewarming party, or in terms of their pleasantness. Date processing and housewarming processing both improved recall compared to pleasantness rating, but date processing and housewarming processing did not produce differences compared to each other. Overall, the current study demonstrates very little evidence of a reproductive processing effect, and nothing approaching the magnitude of previous work on the survival processing effect.
\end{abstract}

Bogdan Kostic

bkostic@missouristate.edu

1 Department of Psychology, University of Pittsburgh, Pittsburgh, PA, USA

2 Department of Psychology, Missouri State University, 901 S. National Ave., Springfield, MO 65897, USA
Keywords Survival processing · Adaptive memory · Reproduction $\cdot$ Mate selection $\cdot$ Evolution

In recent years, the psychological literature has emphasized a functional approach toward understanding cognition and behavior. Specifically, a growing number of researchers have examined ways in which human memory systems were shaped by the kinds of problems that confronted humans in ancestral environments. One of the more influential approaches to this question is to examine how processing information in evolutionarily relevant ways can influence memory. Cognitive psychologists have known for decades that differential information processing can lead to differential performance in retrieval from long-term memory, with deeper (meaning-based) processing leading to better memory (Craik \& Tulving, 1975). Nairne, Thompson, and Pandeirada (2007) were the first to apply this concept to an adaptive context. They hypothesized that because of survival pressures in our evolutionary history survival processing could lead to more effective recall than other deep processing prompts. Nairne et al. tested the possibility of such a survival processing advantage by having participants evaluate words for survival value, value in moving to a foreign country, or pleasantness. Across several different designs and types of memory tests, survival processing consistently led to the best memory performance. Many subsequent studies have replicated this survival processing advantage (Kang, McDermott, \& Cohen, 2008; Kostic, McFarlan, \& Cleary, 2012; Nairne \& Pandeirada, 2008a, 2010; Nairne, Pandeirada, Gregory, \& VanArsdall, 2009; Otgaar, Smeets, \& van Bergen, 2010).

However, basic survival is not the only factor that shapes evolution. Nairne and Pandeirada (2008b) listed other potential types of processing that may have been influenced by evolutionary pressures, including navigation, social exchange, 
kin recognition, and reproduction. Reproduction has an especially important role in evolution, as traits are only adaptive to the extent that they enable an organism to pass on its genes (Miller, 2000).

An example of a reproductive-based adaptation is the asymmetry in male and female reactions to different kinds of infidelity. Buss, Larsen, Westen, and Semmelroth (1992) theorized that because males' reproductive success is more threatened by sexual infidelity (due to uncertain paternity), whereas women's reproductive success is more threatened by emotional infidelity (due to the potential loss of resources), men and women could have adaptively divergent attitudes toward sexual or emotional infidelity. Buss et al. presented participants with a forced choice in which participants selected the more upsetting scenario, given the choice between sexual and emotional infidelity. Men were more likely than women to indicate that sexual infidelity was the more upsetting scenario. These results have been widely replicated (e.g., Sagarin et al., 2012).

There have been several other recent approaches to studying potential reproductive adaptions in memory. Kole and Healy (2011) examined memory for details about people. Participants learned details about familiar people (such as friends and relatives) and unfamiliar people, then compared cued recall of those details across levels of relatedness (e.g., identical twins, first cousins, friends). Participants' memory was better for details about friends or acquaintances compared to genetically related people, and memory was also better for details about people of the opposite sex. Kole and Healy suggested that better memory for unrelated people may be an adaptation for mate selection. Another study with similar implications examined the effect of male vocal pitch of female memory (Smith, Jones, Feinberg, \& Allan, 2012). Smith et al. found that female participants demonstrated better visual object memory when they heard the name of an object spoken in a masculine male voice (but not a masculine female voice). These results also suggest that memory systems are sensitive to information relevant to mate selection. One additional study along these lines was conducted by Smith, Jones, and Allan (2013), who found that women's source memory of men's faces varied depending on women's mating strategies; women pursuing short-term mating strategies showed better memory for exaggerated masculine facial features, while women pursing long-term mating strategies showed better memory for reduced masculine facial features.

In each of the above three studies the authors found support for the possibility that memory systems have evolved sensitivity to information relevant to reproduction, although they did not examine adaptive memory by manipulating the type of processing in the manner of Nairne et al. (2007). By contrast, Sandry, Trafimow, Marks, and Rice (2013) did examine processing sensitivity toward various types of fitness-relevant information. They tested the idea that the survival processing advantage is rooted in a mnemonic advantage for fitness-relevant information. Their experiment was designed to compare survival processing to control conditions (pleasantness and visualization) as well as a wide variety of other fitness-relevant conditions, including separate prompts for both jealousy and mate selection. Rather than compare each of these to control conditions separately, Sandry et al. compared them to the survival processing condition. Because survival processing is one of many kinds of fitness-relevant processing, a significant difference would be unexpected under the adaptive memory paradigm. In both between- and within-subjects designs, their results showed that survival processing significantly outperformed both jealousy and mate selection conditions, and no condition other than survival processing lead to superior recall performance compared to non-fitness-related conditions. The survival processing advantage, they concluded, must be due to some factor other than fitness-relevant processing.

There are several alternative explanations for Sandry et al.'s (2013) results. Perhaps their processing prompts simply failed to activate fitness-relevant processing. Prior research (Butler, Kang, \& Roediger, 2009) has shown that the relevance of the stimuli to the scenario can affect recall. In one experiment, stimuli relevant to a survival scenario were recalled more often after survival processing than robbery processing. But this effect was reversed when the stimuli were relevant to a robbery scenario. In Sandry et al.'s experiments, participants read the following instructions for mate-selection processing:

Experiments 1a and 2

In this task, we would like you to imagine that you are looking for a new partner to have sex with. It is important to identify partners who would sexually satisfy you. We are going to show you a list of words, and we would like you to rate how relevant each of these words would be for you in your search for a sex partner. Some of the words may be relevant and others may not-it's up to you to decide.

Experiment 3

In this task, we would like you to imagine that you are stranded in the grassland of a foreign land, without someone to mate with. Over the next few months, you'll need to find a partner to reproduce and mate with and help you raise children. Below you will find a list of words, and we would like you to rate how relevant each of these words would be for you in this mating situation. Some of the words may be relevant and others may not - it's up to you to decide.

In both conditions, participants were asked to rate a list of nouns. It is possible that objects are not particularly relevant for finding sexual partners, which may inhibit recall according 
to Butler et al. (2009). In mate selection, adjectives describing potential mates are arguably more relevant. However, this line of reasoning is somewhat speculative, and an examination of relevance ratings will be necessary to evaluate how processing nouns compares to processing adjectives in mate-selection scenarios (see the General Discussion).

Our goal for the current study was to investigate a reproductive processing effect in memory using the adaptive memory protocol established by Nairne et al. (2007). In Experiment 1, participants rated adjectives (not nouns) for their relevance in evaluating people in reproductive or nonreproductive scenarios. In Experiment 2 we investigated a reproductive processing effect in memory using an established phenomenon in evolutionary psychology: Buss et al.'s (1992) sex differences in jealousy. In this experiment, we examined potential differences between men and women when rating adjectives for their relevance in detecting different kinds of infidelity (sexual vs. emotional). In Experiment 3, participants rated nouns (not adjectives) for their relevance as gifts in a scenario relevant to reproduction (a romantic date) or a scenario not relevant to reproduction (a housewarming party). Together, these three experiments investigated whether memory systems can be sensitive to several new types of reproductive-based processing.

\section{Experiment 1}

\section{Method}

Participants Based on the .09 partial eta squared $\left(\eta_{\mathrm{p}}{ }^{2}\right)$ effect size obtained from Experiment 1 of Nairne et al. (2007), if a reproductive processing advantage is comparable to Nairne et al.'s survival processing advantage, we would need 102 participants to achieve a power level of .80. Missouri State University undergraduates (122 total; 64 males, 58 females) participated in exchange for course credit or extra credit in a psychology course. Data from one participant were lost due to computer malfunction, but no participants were excluded from analysis.

Materials The stimuli were 30 words (adjectives) that could be used to describe a person, henceforth referred to as "traits." (See Appendix A for the complete list of stimuli.) The stimuli were randomly selected from a list generated by the English Lexicon Project (Balota et al., 2007) using filters to create a list of adjectives between three and 13 letters long and with medium-level word frequency ( $\log$ HAL frequency $M=7.29$, $S D=1.30)$.

Design and procedure The experiment was computer-based and used E-Prime software. Experiment files are available at osf.io/kgr2t. The experiment used a between-subjects design.
After participants provided informed consent, they were randomly assigned to one of three conditions. In each condition, participants viewed 30 traits, one at a time in a unique random order, and provided ratings of those traits according to the scenario in their condition. The traits appeared in the center of the screen and remained there until participants provided their ratings (with an upper limit of 5 seconds).

The three conditions were as follows: In the mate condition $(n=41)$, participants were told to think about a romantic partner in a long-term relationship (real or imaginary) with whom they could envision having children. The participants were told to rate each trait on a scale of 1 to 5 based on how desirable that trait would be in their romantic partner, with 1 meaning they would not like a partner with that trait and 5 meaning they would like a partner with that trait.

In the coworker condition ( $n=39$ ), participants were told to think about a coworker (real or imaginary) they could envision themselves working closely alongside in a long-term career. The participants were told to rate each trait on a scale of 1 to 5 based on how much they would like their coworker to exhibit that trait, with 1 meaning they would not like a coworker with that trait and 5 meaning they would like a coworker with that trait.

In the pleasantness condition $(n=42)$, participants were told to rate how pleasant they found each trait on a scale of 1 to 5, with 1 being very unpleasant and 5 being very pleasant.

Participants in all conditions were given an example trial in which they rated the example trait "happy." After participants rated all 30 traits, they performed math problems for 2 minutes as a distractor task. Then, they were given 10 minutes to recall as many traits as they could from the rating task in any order by typing them into the computer. After they finished they were debriefed and thanked for participating.

\section{Results and discussion}

Data are available at osf.io/kgr2t. The data of primary interest ${ }^{1}$ are the number of traits correctly recalled from each processing condition (see Table 1 and Fig. 1). In a planned analysis, a 3 (processing condition: mate vs. coworker vs. pleasantness) $\times$ 2 (participant sex: male vs. female) between-groups ANOVA showed a main effect of processing condition, $F(2,116)=$ $3.53, p=.03, \mathrm{n}_{\mathrm{p}}{ }^{2}=.06$. There was no significant main effect of participant sex, $F(1,116)=1.30, p=.26$, and no interaction between processing condition and sex, $F(2,116)=0.76, p=$ 47. We used independent-samples $t$ tests for pairwise comparisons between the processing conditions. A comparison between the mate condition and the pleasantness condition

\footnotetext{
${ }^{1}$ We allowed for typos as potential instances of correct recall or intrusions, but scoring this type of data is obviously subject to interpretation. We conducted analyses on stringent as well as more permissive coding schemes, and none of them changed the overall pattern of results.
} 
Table 1 Descriptive statistics for Experiment 1

\begin{tabular}{|c|c|c|c|c|c|c|c|c|c|}
\hline \multirow[b]{2}{*}{ Processing condition } & \multirow[b]{2}{*}{$n$} & \multicolumn{2}{|l|}{ Recall } & \multicolumn{2}{|c|}{ Intrusions } & \multicolumn{2}{|c|}{ Ratings } & \multicolumn{2}{|c|}{ Response Times (ms) } \\
\hline & & $M$ & $S D$ & $M$ & $S D$ & $M$ & $S D$ & $M$ & $S D$ \\
\hline Mate & 41 & 10.63 & 4.33 & 5.07 & 4.34 & 2.95 & 0.24 & $2,127.63$ & 409.12 \\
\hline Coworker & 39 & 9.64 & 4.85 & 4.56 & 5.01 & 2.98 & 0.29 & $1,997.67$ & 402.01 \\
\hline Pleasantness & 42 & 8.24 & 3.21 & 3.38 & 3.61 & 3.16 & 0.22 & $1,896.75$ & 424.16 \\
\hline
\end{tabular}

showed significantly higher recall of traits processed in the mate condition, $t(81)=2.87, p<.01$, Cohen's $d=0.64$. However, there was no significant difference in the recall of traits processed in the mate and coworker conditions, $t(78)=$ $0.97, p=.34$, nor was there a difference between the coworker and pleasantness conditions, $t(65.55)=1.54, p=.13$.

We also conducted unplanned analyses on the intrusionsrecall of words that were not on the study list. Intrusions were problematic to code because the recall task was computerbased and it did not allow participants to modify their responses once they were entered. As a result, participants often would type in the same word several times apparently in an attempt to spell it correctly, or they might even type in random things that did not appear to be recall attempts (e.g., word fragments or random thoughts like "i dont know"). We strove to only code attempts as intrusions if they appeared to be traits that could conceivably be used to describe a person and we only counted an intrusion once if it was entered multiple times (even with different spellings). Intrusions coded thusly, a oneway ANOVA showed no significant difference in the intrusion rate across processing conditions, $F(2,119)=1.66, p=.19$ *see Table 1).

Turning to the secondary data of interest, we conducted planned comparisons on the average ratings and response times from each processing condition (see Table 1). A one-way between-groups ANOVA on the ratings showed a significant difference, $F(2,119)=8.91, p<.001, \mathrm{\eta}_{\mathrm{p}}{ }^{2}=.13$. In pairwise comparisons, ratings in the mate condition were not

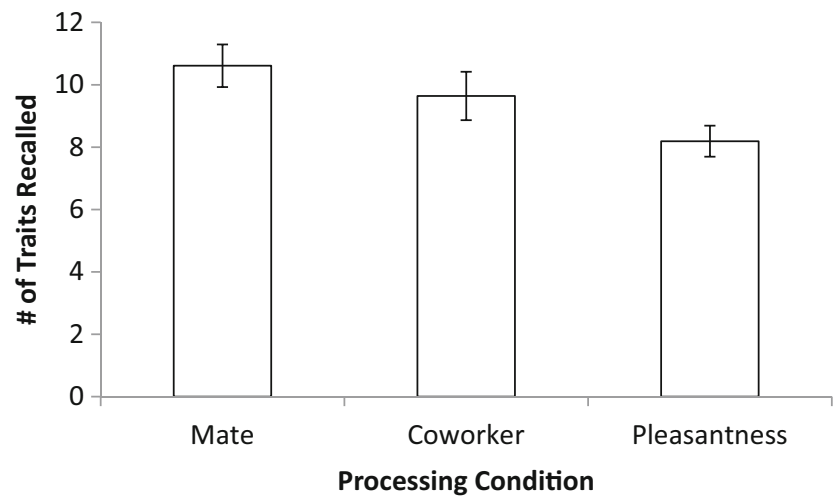

Fig. 1 Average number of traits recalled from each processing condition in Experiment 1. Error bars depict standard error of the mean. The $y$-axis is truncated for clarity; the maximum possible number of traits that could be recalled is 30 significantly lower than in the coworker condition, $t(78)=0.43$, $p=.67$. Ratings in the mate condition were significantly lower than in the pleasantness condition, $t(81)=4.27, p<.001$, Cohen's $d=0.94$, and ratings in the coworker condition were also lower than those in the pleasantness condition, $t(79)=$ $3.28, p<.01$, Cohen's $d=0.73$. The same type of ANOVA on the response times also showed a significant difference between conditions, $F(2,119)=3.27, p=.04, \eta_{\mathrm{p}}{ }^{2}=.05$, with response times being comparable in the mate condition and in the coworker condition, $t(78)=1.43, p=.16$, but with response times being significantly faster in the pleasantness condition compared to the mate condition, $t(81)=2.52, p=.01$, Cohen's $d=0.55$, but not compared to the coworker condition, $t(79)=1.10, p=.28$.

In an unplanned items analysis we explored the relationship between relevance ratings and recall by calculating Pearson correlations between average ratings and recall for each stimulus in each processing condition. There were no significant correlations in any condition, mate: $r(29)=-.14, p=.46$; coworker: $r(29)=-.31, p=.10$; pleasantness: $r(29)=-.12, p$ $=.54$. However, ratings of stimuli in each condition correlated very highly with ratings of the stimuli in the other conditions, mate versus coworker: $r(29)=.97, p<.001$; mate versus pleasantness: $r(29)=.98, p<.001$; coworker versus pleasantness: $r(29)=.97, p<.001$.

To further examine whether the relevance ratings could influence the recall pattern, we conducted a follow-up between-subjects ANCOVA that included average ratings as a covariate. The analyses showed that ratings did not significantly affect recall, $F(1,118)=0.50, p=.48$. The main effect of processing condition remained significant, $F(2,118)=$ $3.66, p=.03, \mathrm{\eta}_{\mathrm{p}}{ }^{2}=.06$. This suggests the recall results are likely not being driven by the relevance ratings.

The results of Experiment 1 provided some evidence of a reproductive processing advantage in memory, in that processing traits of potential romantic partners produced slightly better recall than the control condition of pleasantness processing. But processing in the mate condition did not produce better recall than processing in the coworker condition, which is the more stringent criterion (comparable to previous research) of whether evolutionary factors shaped memory systems. Reaction times varied across conditions, but faster reaction times do not necessarily prevent recall superiority (e.g., Experiment 2 of Kang et al., 2008). Nevertheless, the 
overall pattern of recall in Fig. 1 suggests the potential for a reproductive processing advantage, perhaps under different conditions. In Experiment 2 we modified our experimental procedure in order to base the processing task on a wellestablished paradigm in evolutionary psychology: sex differences in jealousy. Experiment 2's processing conditions involved asking participants to suspect their partners were being unfaithful (either sexually or emotionally), and participants were tasked with rating various traits of their partners in terms of how helpful they would be at detecting infidelity. Utilizing a more established theoretical paradigm in evolutionary psychology may help detect a reproductive processing effect in memory, and another benefit is that the task is similar to several employed by Sandry et al. (2013), such as their jealousy and infidelity conditions. Experiment 2 will therefore further explore whether rating adjectives is a more relevant task than rating nouns for reproductive processing.

\section{Experiment 2a}

\section{Method}

Participants We conducted a power analysis using effect size estimates from previous studies in both the adaptive memory and jealousy paradigms. Past experiments comparing survival processing to control conditions in within-subjects designs (Kang et al., 2008; Nairne \& Pandeirada, 2008a, 2010; Nairne, Pandeirada, \& Thompson, 2008; Nairne et al., 2007; Otgaar et al., 2010) have yielded large effect sizes averaging $d=$ 1.25. However, the relevant effect for this study is an interaction between participant sex and jealousy prompt. As such, a more accurate estimate of expected effect size would come from the jealousy literature. Sagarin et al. (2012) found an average effect of $g^{*}=.258$ for studies in the Buss jealousy paradigm that used continuous measures. This is interpreted as a small effect size, conservatively equivalent to a $d$ of the same value. Harris (2003) found an average effect of 1.00 (log-odds ratio), which is considered a medium-sized effect, equivalent to $d=.55$. Schützwohl and Koch (2004) obtained a fairly large effect for the relevant interaction with a Cohen's $d$ of .75. Using these values to provide context for the present study, we opted to estimate a medium effect size of $d=.5$. With this effect size, and power set at .80 , a sample size of 64 participants was required to detect any significant effect, if one is present.

Participants were 93 (62 female, 31 male) undergraduate psychology students at Missouri State University who participated in exchange for course credit.

Materials Stimuli consisted of 60 adjectives that could be used to describe people in terms of physical or personality traits. See Appendix A for the complete list of stimuli. The stimuli were randomly selected from a list generated by the
English Lexicon Project (Balota et al., 2007) using filters to create a list of adjectives between three and 13 letters long and with medium-level word frequency ( $\log$ HAL frequency $M=$ $6.09, S D=2.20$ ).

Design and procedure The experiment was computer-based and used E-Prime software. Experiment files are available at osf.io/kgr2t. The experiment used a within-subjects design. Participants were given 10 seconds to rate each trait in one of three conditions. As in Experiment 1, the stimuli appeared onscreen until the participant rated the trait (with a 10-second cutoff). Participants were given more time than in Experiment 1 because the prompts on each trial in Experiment 2 were longer (see prompts below). ${ }^{2}$ The order of stimulus presentation was randomized for each participant.

Participants were given a total of three processing prompts to evoke different kinds of processing of the stimuli. The processing conditions were presented in randomly ordered blocks. Stimuli were randomly assigned to conditions so that the same trait could have been rated in one condition for one participant and another condition for another participant.

The two experimental conditions were designed to provoke sexual and emotional jealousy. In the sexual jealousy condition, participants were told to imagine their partner in a committed relationship. They were given a series of traits to rate in accordance with how helpful they would be in predicting whether or not a partner would commit sexual infidelity:

Imagine your partner is [trait]. On a scale of 1 (very unhelpful) to 5 (very helpful), how helpful is this trait in judging whether or not they will have sex with someone else?

In the emotional jealousy condition, participants received the same instruction as the sexual jealousy condition, except that the goal in the rating task was to predict emotional infidelity:

Imagine your partner is [trait].On a scale of 1 (very unhelpful) to 5 (very helpful), how helpful is this trait in judging whether or not they will fall in love with someone else?

In the control condition, participants rated the traits for pleasantness. See Appendix B for complete instructions in each condition.

Each processing block was preceded with instructions and one practice rating trial using the word secretive. After

\footnotetext{
${ }^{2}$ We did not know in advance how long participants would need to read the longer prompt, so we increased the maximum amount of time each stimulus could appear. In spite of this concern, Tables 1,3 , and 4 show that average response times were below 5 seconds in all three experiments.
} 
completing the three processing blocks, participants solved math problems for 2 minutes as a distractor task. Participants then completed a surprise recall task for 10 minutes, in which they were instructed to type in as many of the rated words as they could remember. After the recall task, all participants completed a forced-choice task in a direct replication of Buss et al. (1992):

For this task, please think of the romantic relationship you pictured earlier. Which of the scenarios caused you to be more upset?

1. Imagining my partner falling in love with someone else (Press 1)

2. Imagining my partner enjoying passionate sex with someone else (Press 2)

Pick the one that most applies.

Finally, participants were asked if they had ever been in a serious, committed romantic relationship, and if they were currently in such a relationship. When participants were finished, the experimenter debriefed them.

\section{Results and discussion}

Data are available at osf.io/kgr2t. It is first important to determine whether the current results replicate Buss et al. (1992) with regard to sex differences in jealousy, otherwise there would be no basis to suspect differences in the processing conditions. The analysis of the forced-choice question about infidelity (whether sexual or emotional infidelity would be more upsetting) replicated Buss et al.'s finding: men were more likely than women to report that sexual infidelity would be more upsetting $\chi^{2}(1)=18.19, p<.001$ (see the left half of Fig. 2.) The same basic pattern holds when participants are separated by their relationship histories; men are more likely than women to report that sexual infidelity would be more distressing, regardless of whether they are currently in a relationship, previously in a relationship, or have never been in a relationship. In contrast, Buss et al. found that men who had been in a relationship were more likely to indicate that sexual infidelity was more distressing, but the same effect did not appear for men who had not been in a relationship. However, in the current study, there were occasionally fewer than five participants in each category of relationship history (see Table 2), which makes it difficult to draw meaningful conclusions about relationship histories. All subsequent analyses combine all participants, regardless of their relationship histories.

As in Experiment 1, the data of primary interest are the number of traits correctly recalled from each processing condition (see Table 3 and Fig. 3). In a planned analysis, we conducted a 3 (processing condition: sexual jealousy vs. emotional jealousy vs. pleasantness) $\times 2$ (participant sex: male vs . female) mixed-factors ANOVA with repeated measures on the first factor. There was no significant main effect of processing condition, $F(2,182)=0.88, p=.42$, and no significant main effect for sex, $F(1,91)=.02, p=.88$. The analysis revealed no significant interaction, $F(2,182)=0.10, p=.90$. Contrary to the reproductive advantage hypothesis, no processing condition produced superior recall over any other condition. Additionally, recall was comparable for males and females. Intrusions were coded the same way as for Experiment 1, and the average number of intrusions for each participant was $6.82(S D=5.06)$.

In an exploratory analysis, we conducted a slightly more complex ANOVA in which we added participants' responses to the jealousy question as a between-subjects factor. This 3 (processing condition: sexual jealousy vs. emotional jealousy vs. pleasantness) $\times 2$ (participant sex: male vs. female) $\times 2$ (jealousy response ${ }^{3}$ : emotional vs. sexual) mixed-factors ANOVA showed the same lack of main effect of processing and sex as above, but there was a significant interaction between participant sex and jealousy response, $F(1,89)=$ 4.86, $p=.03, \mathrm{\eta}_{\mathrm{p}}{ }^{2}=.05$, in which men who responded that emotional infidelity is worse tended to recall more traits than men who responded that sexual infidelity is worse, while women who responded that sexual infidelity is worse tended to recall more traits than women who responded that emotional infidelity is worse (see Fig. 4.) The three-way interaction between processing condition, sex, and jealousy response was not significant, $F(2,178)=0.52, p=.60$.

To examine differences in ratings and response times across processing conditions, we conducted one-way repeated-measures ANOVAs. See Table 3 for descriptive statistics of ratings and response times. There was a significant difference in ratings between the processing conditions, $F(2,184)$, = 5.01, $p<.01, \mathrm{\eta}_{\mathrm{p}}{ }^{2}=.05$. Post hoc tests revealed significant differences in ratings between sexual jealousy and pleasantness processing, $t(92)=2.78, p<.01, d=0.41$, as well as between emotional jealousy and pleasantness processing, $t(92)=2.04, p=.04, d=0.26$, indicating that participants provided higher ratings to words in both jealousy conditions compared to the Pleasantness condition. In other words, participants' ratings were higher for relevance to detecting infidelity than for pleasantness. There was no significant difference in ratings between sexual jealousy and emotional jealousy conditions, $t(92)=1.27, p=.21$. The same type of ANOVA revealed significant differences in response times between the processing conditions, $F(2,184)=52.75, p<.001$, $\eta_{\mathrm{p}}{ }^{2}=.36$. Post hoc tests showed that participants responded faster in the pleasantness condition than both the sexual

\footnotetext{
${ }^{3}$ Emotional $=$ responding that emotional infidelity is more upsetting. Sexual = responding that sexual infidelity is more upsetting.
} 


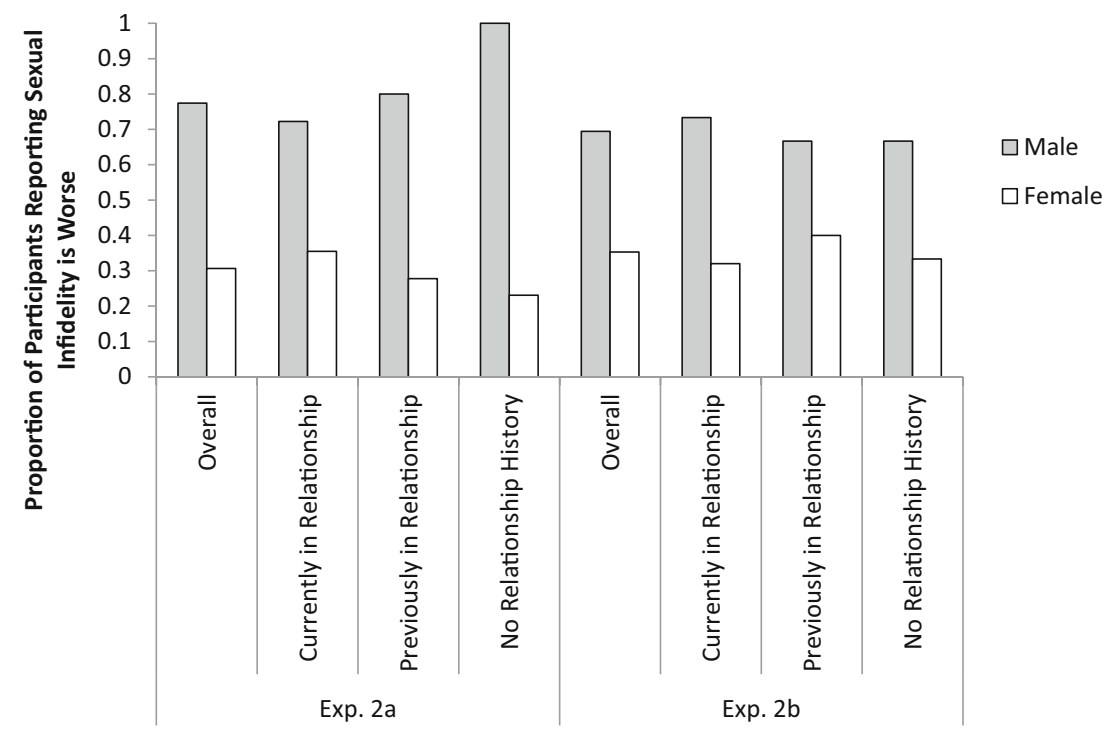

Fig. 2 Replication of the forced-choice task from Buss et al. (1992), split by relationship history in Experiments 2a and 2b

processing condition, $t(92)=9.69, p<.001, d=0.86$, and the emotional processing condition, $t(92)=9.45, p<.001, d=$ 0.84 . There was no significant difference in response times between sexual processing and emotional processing conditions, $t(92)=0.39, p=.70$. A likely explanation for the pattern of faster response times in the pleasantness condition is simply that the rating prompt on the screen was much shorter in the pleasantness condition than in either jealousy processing condition and therefore took less time to read.

In an unplanned items analysis we explored the relationship between relevance ratings and recall by calculating Pearson correlations between average ratings and recall for each stimulus in each processing condition. As in Experiment 1, we found no significant correlation between ratings and recall of each stimulus in any condition, sexual processing: $r(59)=.13$, $p=.31$, emotional processing: $r(59)=.08, p=.57$, and pleasantness: $r(59)=.05, p=.71$. Similar to Experiment 1 , we found some significant correlations between ratings of stimuli

Table 2 Frequencies for relationship history by sex in Experiment 2

\begin{tabular}{lllll}
\hline Exp. & Relationship history & Male & Female & Total \\
\hline 2a & Current relationship & $18(13)$ & $31(11)$ & $49(24)$ \\
& Past relationship & $10(8)$ & $18(5)$ & $28(13)$ \\
& No relationship & $3(3)$ & $13(3)$ & $16(6)$ \\
& Total & $31(24)$ & $62(19)$ & $93(43)$ \\
bb & Current relationship & $15(11)$ & $25(8)$ & $40(19)$ \\
& Past relationship & $12(8)$ & $25(10)$ & $37(18)$ \\
& No relationship & $9(6)$ & $18(6)$ & $27(12)$ \\
& Total & $36(25)$ & $68(24)$ & $104(49)$ \\
\hline
\end{tabular}

Note. The number in parentheses indicates the number of participants who responded that sexual infidelity would be worse than emotional infidelity in sexual versus emotional processing conditions, $r(59)=.83$, $p<.001$, and sexual versus pleasantness processing conditions, $r(59)=-.39, p<.001$. However, unlike Experiment 1 , the correlations did not appear in all comparisons; ratings in the emotional versus pleasantness conditions were not significantly correlated, $r(59)=-.23, p=.08$. Another difference with Experiment 1 was that correlations with pleasantness ratings were negative (possibly because traits that are good for infidelity detection are not pleasant).

In another unplanned analysis, we ran a multilevel model analysis to examine any potential effects of average ratings as an adjustor of recall. Fit indices for the random intercepts model were AIC $=1,188.34$ and $\mathrm{BIC}=1,199.28$. Ratings were a significant predictor of recall compared to the random intercepts only model, AIC $=1,182.17, \mathrm{BIC}=1,196.69, p<$ .01 . However, after controlling for ratings, adding processing condition and sex to the model as fixed effects did not contribute enough variance to significantly improve the model, $\mathrm{AIC}=1,186.80, \mathrm{BIC}=1,212.22, p=.71$. These results indicated that there were still no significant differences in recall between processing conditions when including the effect of relevance ratings.

Experiment 2a failed to show any evidence that processing traits for their value in detecting sexual or emotional infidelity produced differences in recall between each other or compared to a control condition of pleasantness processing. There was some evidence to suggest that participants' memory may be sensitive to the type of processing condition depending on what type of infidelity (emotional or sexual) they perceive to be worse. The results suggested that men who are sensitive to emotional infidelity recall more than men who are sensitive to sexual infidelity, while women who are sensitive to sexual infidelity cues recall more than women who are sensitive to emotional infidelity. In other 
Table 3 Descriptive statistics for Experiments 2a and 2b

\begin{tabular}{|c|c|c|c|c|c|c|c|}
\hline \multirow[b]{2}{*}{ Exp. } & \multirow[b]{2}{*}{ Processing condition } & \multicolumn{2}{|c|}{ Recall } & \multicolumn{2}{|c|}{ Ratings } & \multicolumn{2}{|c|}{ Response times (ms) } \\
\hline & & $M$ & $S D$ & $M$ & $S D$ & $M$ & $S D$ \\
\hline \multirow[t]{3}{*}{$2 \mathrm{a}$} & Sexual jealousy & 2.74 & 1.87 & 2.82 & 0.61 & $3,046.32$ & $1,012.38$ \\
\hline & Emotional jealousy & 2.81 & 2.18 & 2.75 & 0.62 & $3,010.49$ & 975.83 \\
\hline & Pleasantness & 2.42 & 2.13 & 2.61 & 0.42 & $2,304.18$ & 682.08 \\
\hline \multirow[t]{3}{*}{$2 b$} & Sexual jealousy & 4.65 & 2.70 & 2.45 & 0.61 & $2,678.13$ & $1,037.33$ \\
\hline & Emotional jealousy & 4.55 & 2.63 & 2.47 & 0.58 & $2,724.09$ & 970.09 \\
\hline & Pleasantness & 4.35 & 2.53 & 3.00 & 0.35 & $1,824.06$ & 487.93 \\
\hline
\end{tabular}

words, sensitivity to processing condition only appeared for participants who are atypical (for their sex) in terms of their jealousy. However, these analyses were exploratory and should be interpreted with caution. It is also worth noting that participants with sex-atypical jealousy responses were in the minority; only seven men responded that emotional infidelity is worse, and only 19 women responded that sexual infidelity is worse (see Table 2).

Overall, Fig. 3 shows unusually low rates of recall compared to typical survival processing effects (e.g., Nairne \& Pandeirada, 2008b), and even the reproductive processing conditions in Experiment 1, although they were somewhat comparable to the levels demonstrated in Sandry et al. (2013). One potential reason for the low recall performance could be due to word frequency effects. It has long been established that lower frequency words are more difficult to recall than higher frequency words (Hall, 1954), and Experiment $2 \mathrm{a}$ used stimuli whose log HAL frequency $(M=$ $6.09, S D=2.20$ ) were somewhat lower in word frequency than those of Experiment $1(M=7.29, S D=1.30)$ and a benchmark study such as Nairne et al. (2007; $M=9.05, S D$ $=1.31$ ). So to evaluate the possibility that low recall rates were due to low word frequency, Experiment $2 b$ was conducted with a revised set of stimuli. Experiment $2 b$ also further examined the unexpected interaction between processing condition and jealousy response. Experiment $2 b$ used the same methods and design as Experiment 2a, but the stimuli were traits with a higher average word frequency.

\section{Experiment 2b}

\section{Method}

Participants The same power analysis from Experiment 2a suggested that a sample size of 64 would be needed to achieve a power level of .80. Participants were 104 (68 female, 36 male) undergraduate psychology students at Missouri State University who participated in exchange for course credit. Data from three participants were lost due to computer malfunction, but no participants were excluded from analysis.

Materials Stimuli consisted of 60 adjectives that could be used to describe people in terms of physical or personality traits (see Appendix A for the complete list of stimuli). The stimuli were generated by research assistants who were blind to the hypothesis and tasked only with thinking of adjectives that could be used to describe people. The experimenters then randomly selected the final set to be used in the experiment. The stimuli ranged between three and 12 letters in length. The English Lexicon Project (Balota et al., 2007) reports the mean $\log$ HAL frequency of the stimuli in this experiment was 8.47 $(S D=1.25)^{4}$

Design and procedure Experiment files are available at osf.io/kgr2t. Except for the different stimuli, all other aspects of the design and procedure for the current experiment were identical to those of Experiment 2a.

\section{Results and discussion}

Data are available at osf.io/kgr2t. As in Experiment 2a, it is first important to determine whether the current results replicate Buss et al.'s (1992) effect of sex differences in jealousy. As in Experiment 2a, the effect did replicate; men were more likely than women to report that sexual infidelity is more distressing, $\chi^{2}(1)=11.02, p<.01$, and the same basic pattern held across all levels of relationship experience. See the right half of Fig. 2.

As in Experiment 2a, the primary data of interest are the number of traits recalled from each processing condition, and the main (planned) analysis is a 3 (processing condition: sexual vs. emotional vs. pleasantness) $\times 2$ (participant sex: male vs. female) mixed-factors ANOVA with repeated measures on the first factor. See Table 3 and Fig. 3 for descriptive statistics. Again, the main effect of processing condition was not significant, $F(2$,

\footnotetext{
${ }^{4}$ The frequencies for nosey and quirky were not available in the English Lexicon Project database.
} 


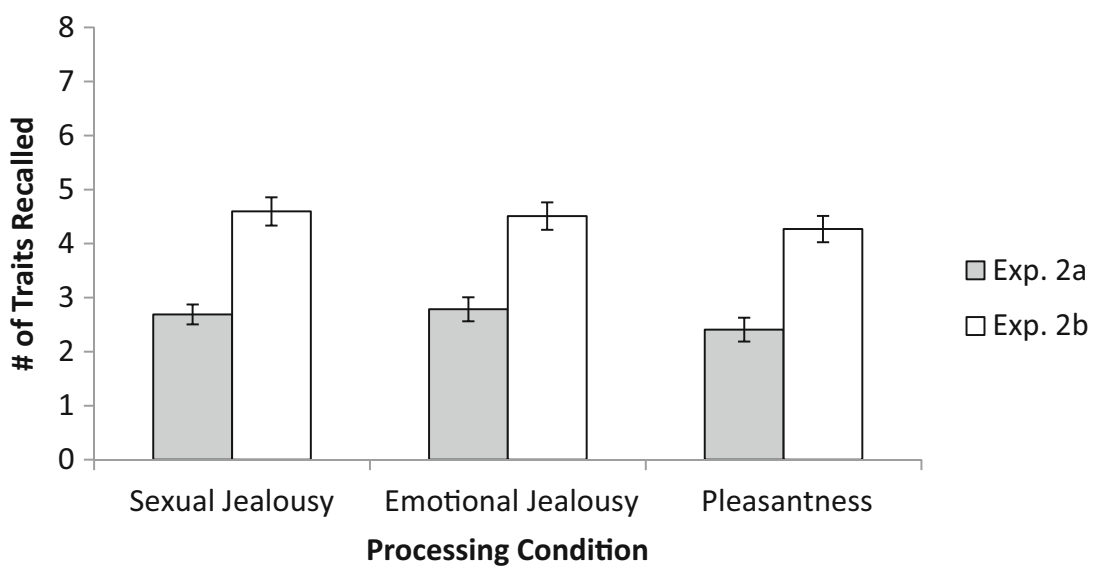

Fig. 3 Average number of traits recalled from each processing condition in Experiments 2a and 2b. Error bars depict standard error of the mean. The $y$ axis is truncated for clarity; the maximum possible number of traits that could be recalled in each condition is 20

204) $=0.72, p=.49$, which shows that recall was comparable across all conditions. However, one difference between the Experiment $2 \mathrm{a}$ and Experiment $2 \mathrm{~b}$ is that in Experiment $2 \mathrm{~b}$ there was a significant main effect of sex, $F(1,102)=4.03, p=.05, \eta_{\mathrm{p}}{ }^{2}=.04$, in which women recalled slightly more traits than men, although the interaction between sex and processing condition was not significant, $F(2,204)=0.20, p=$ .82 (see Fig. 5). Intrusions were coded the same way as for Experiments 1 and $2 \mathrm{a}$, and the average number of intrusions for each participant was $4.38(S D=3.73)$.

We also conducted the same exploratory analysis as in Experiment $2 \mathrm{a}$ in which we included responses to the jealousy question in a 3 (processing condition: sexual vs. emotional vs. pleasantness $) \times 2$ (participant sex: male vs. female) $\times 2$ (jealousy response ${ }^{5}$ : emotional vs. sexual) mixed-factors ANOVA. We obtained slightly different results than in Experiment 2a; in the current experiment, the interaction between sex and jealousy response was not significant, $F(1,100)=2.47, p=.12$, but the three-way interaction between processing condition, sex, and jealousy response was significant, $F(2,200)=5.74, p<.01, \mathrm{n}_{\mathrm{p}}{ }^{2}=.05$. As Fig. 5 shows, the three-way interaction appears to depict that women recalled more than men in the sexual processing condition only when they responded that sexual infidelity is worse, but women recalled more than men in the emotional processing condition only when they responded that emotional infidelity is worse. In other words, women's memory systems may be sensitive to the type of processing depending on their "hot button" type of jealousy, but men do not show this sensitivity.

To examine differences in ratings and response times across processing conditions, we again conducted one-way

\footnotetext{
5 Emotional $=$ responding that emotional infidelity is more upsetting. Sexual $=$ responding that sexual infidelity is more upsetting.
}

repeated-measures ANOVAs. See Table 3 for descriptive statistics of ratings and response times. There was a significant difference in ratings between the processing conditions, $F(2$, 206), $=46.20, p<.001, \mathrm{n}_{\mathrm{p}}{ }^{2}=.31$. Post hoc tests revealed significant differences in ratings between sexual processing and pleasantness processing, $t(103)=7.87, p<.001, d=$ 1.11 , as well as between emotional processing and pleasantness processing, $t(103)=8.03, p<.001, d=1.12$, indicating that participants provided lower ratings to words in both the sexual and emotional processing conditions compared to the pleasantness condition. There was no significant difference in ratings between sexual jealousy and emotional jealousy conditions, $t(103)=0.34, p=.74$. This pattern is different from what appeared in Experiment 2a, likely due to the fact that Experiment $2 b$ used a different set of stimuli that may have been less relevant to detecting infidelity, or more pleasant, or both. The same type of ANOVA revealed significant differences in response times between the processing conditions, $F(2,206)=64.95, p<.001, \mathrm{n}_{\mathrm{p}}{ }^{2}=.39$. Post hoc tests showed that participants responded faster in the pleasantness condition than both the sexual processing condition, $t(103)=$ $9.81, p<.001, d=1.06$, and the emotional processing condition, $t(92)=12.06, p<.001, d=1.18$. There was no significant difference in response times between sexual processing and emotional processing conditions, $t(103)=0.45, p=.66$ (again, likely due to the shorter rating prompt in the pleasantness condition).

In an unplanned items analysis, we explored the relationship between relevance ratings and recall by calculating Pearson correlations between average ratings and recall for each stimulus in each processing condition. As in Experiments 1 and 2a, we found no significant correlation between ratings and recall of each stimulus in any condition, sexual processing: $r(59)=-.07, p=.62,0$ emotional processing: $r(59)=.05, p=.73$, and pleasantness: $r(59)=-.14$, $p=.31$. Similar to previous experiments, we found significant 


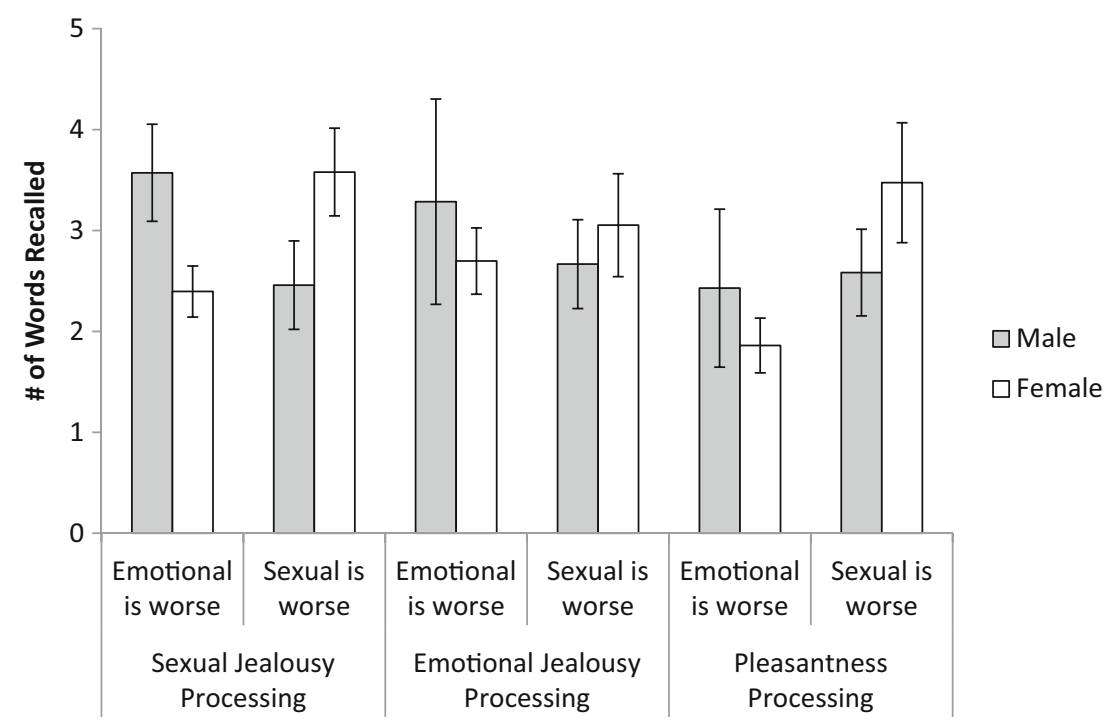

Fig. 4 Average number of traits recalled from each processing condition in Experiments 2a, separated by participant sex (male vs. female) and whether participants indicated that emotional or sexual infidelity would

correlations between ratings of stimuli in sexual versus emotional processing conditions, $r(59)=.95, p<.001$, sexual versus pleasantness processing conditions, $r(59)=-.78, p<$ .001 , and emotional versus pleasantness conditions, $r(59)$ $=-.75, p<.001$. All three of the comparisons were significantly correlated, unlike in Experiment 2a, but they showed the same trend of negative correlations between cheater detection processing and pleasantness ratings (possibly because traits that are good for infidelity detection are not pleasant).

As in Experiment 2a, we conducted an unplanned multilevel model analysis to examine the potential effect of average be worse. Error bars depict standard error of the mean. The $y$-axis is truncated for clarity; the maximum possible number of traits that could be recalled in each condition is 20

ratings as an adjuster of recall. Fit indices for the random intercepts model were $\mathrm{AIC}=1,418.87$ and $\mathrm{BIC}=1,430.04$. Unlike in Experiment 2a, the addition of ratings into the model did not significantly improve the model, compared to the random intercepts only model, AIC $=1,420.61, \mathrm{BIC}=1,435.51$, $p=.61$. The addition of processing condition and sex also did not significantly improve the model, $\mathrm{AIC}=1,421.64, \mathrm{BIC}=$ $1,447.71, p=.17$. In other words, neither the different processing conditions nor the relevance ratings had any significant effect on recall.

Like Experiment 2a, Experiment 2b showed unexpected interactions between the processing condition

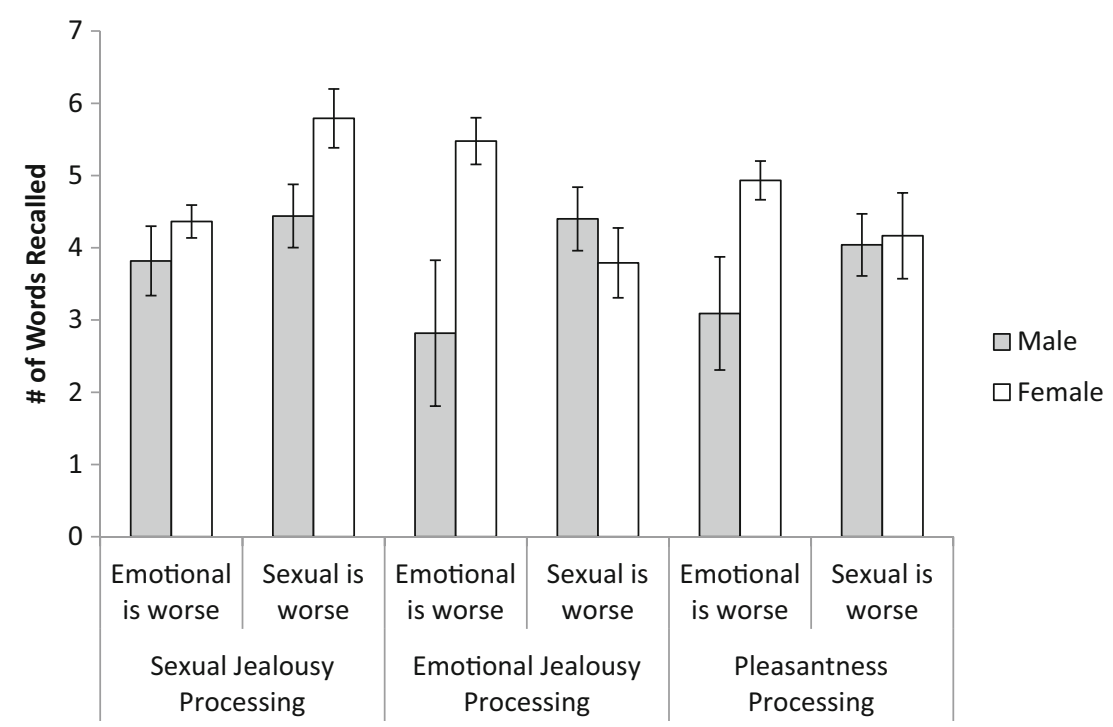

Fig. 5 Average number of traits recalled from each processing condition in Experiments 2b, separated by participant sex (male vs. female) and whether participants indicated that emotional or sexual infidelity would be worse. Error bars depict standard error of the mean. The $y$-axis is truncated for clarity; the maximum possible number of traits that could be recalled in each condition is 20 
and jealousy response, but only when participant sex was also considered, and the unexpected interactions in Experiment $2 \mathrm{a}$ were different than the unexpected interactions in Experiment 2b. Unlike in Experiment 2a, Experiment $2 \mathrm{~b}$ showed that women tended to recall more overall than men, that women may show some sensitivity to processing condition depending on what type of infidelity they perceive to be worse, and that men tend not to show this sensitivity. Once again, these comparisons were not planned, the results were not predicted, and there were small numbers of participants in some conditions (with only 11 men responding that emotional infidelity is worse). The results do not follow exactly the same pattern as in Experiment 2a (perhaps due to the different stimuli in each experiment), but they still suggest that participant sex, jealousy, or both may influence recall performance. Sexual orientation of the participants may have also been involved, but we neglected to ask participants about sexual orientation in this experiment. We did ask participants from the same population to report sexual orientation in Experiment 3, and relatively few people reported being nonheterosexual, so it is unlikely (but possible) that the low number of nonheterosexual participants in this population could be driving any effect. (Also, see prior research showing that homosexual participants tend to respond similarly to heterosexual participants on measures of sex-based differences; Bailey, Gaulin, Agyei, $\&$ Gladue, 1994). Overall, the results hint at the possibility that under some conditions memory systems may have adapted sensitivity to reproductively relevant information.

Experiments 1 and 2 differed from previous work on the adaptive memory paradigm, not only on the type of processing conditions (reproductive-based instead of survival-based) but also on the type of stimuli. The current experiments thus far have used adjectives, while previous work has used nouns. Because adjectives and nouns may differ in terms of qualities such as concreteness and visualizability, processing and recall of the stimuli may differ as well. Our goal in Experiment 3 was to address this possibility by using nouns as stimuli in a reproductive-based processing scenario. Importantly, we created a scenario in which the noun stimuli would be very relevant to reproductive success, avoiding the problem potentially encountered by Sandry et al. (2013). In Experiment 3 participants rated the appropriateness of a gift in a reproductive scenario (a romantic date) or a nonreproductive scenario (a housewarming party). As with Experiments 1 and 2, we are investigating whether reproductive-based processing provides any kind of benefit to memory compared to nonreproductive processing.

\section{Experiment 3}

\section{Method}

Participants Based on the same power analysis we used for Experiment 1, we expected a sample size of 102 participants would produce a power level of .80 . Missouri State University undergraduates (112 total; 46 males, 66 females) participated in exchange for course credit or extra credit in a psychology course. One hundred participants self-identified as heterosexual, five participants self-identified as homosexual, four participants self-identified as bisexual, two participants selfidentified as "other," and one participant responded "prefer not to say." One participant was excluded from analysis for failing to follow instructions.

Materials The stimuli were 32 words (nouns) that could conceivably be given as gifts in a variety of contexts. The complete list of stimuli is in Appendix A. The stimuli were generated by research assistants who were blind to the hypothesis and tasked only with thinking of potential gifts that could be given on a variety of occasions. The experimenters then randomly selected the final set to be used in the experiment. The stimuli ranged between three and nine letters in length. The English Lexicon Project (Balota et al., 2007) reports that the mean $\log$ HAL frequency of the stimuli in this experiment was $8.55(S D=1.72)$

Design and procedure Experiment files are available at osf.io/kgr2t. The experiment was computer-based and used E-Prime software. The experiment used a between-subjects design. After participants provided informed consent, they were randomly assigned to one of three conditions. In each condition, participants viewed 32 traits, one at a time, in a unique random order and provided ratings of those traits according to the scenario in their condition. The traits appeared in the center of the screen until participants provided their ratings (with an upper limit of 5 seconds).

The three conditions were as follows: In the date condition $(n=38)$, participants received the following instructions:

Imagine that a guy is going on a romantic date with a girl and he wants to bring her a gift. The gift should be something nice that shows how much he likes her. We are going to show you a list of potential gifts and you will rate how good each gift would be for a romantic date. Some of the gifts may be good and some may be bad-it's up to you to decide. 
In the housewarming condition $(n=37)$, participants received the following instructions:

Imagine that somebody is going to a housewarming party for some friends and that person wants to bring a gift for them. The gift should be something nice so that the friends will be happy in their new home. We are going to show you a list of potential gifts, and you will rate how good each gift would be for the friends' housewarming party. Some of the gifts may be good and some may be bad-it's up to you to decide.

The participants were told to rate each gift on a scale of 1 to 5 based on how good each gift would be in that context, with 1 meaning the gift is very bad and 5 meaning the gift is very good.

In the pleasantness condition $(n=37)$, participants were told to rate how pleasant they find each word on a scale of 1 to 5 , with 1 being very unpleasant and 5 being very pleasant.

Participants in all conditions were given an example trail in which they rated the word chocolate. After participants rated all 32 words, they performed math problems for 2 minutes as a distractor task. Then, participants were given 10 minutes to recall as many words as they could from the rating task in any order by typing them into the computer. After they were finished, participants self-reported their sexual orientations before being debriefed and thanked for participating.

\section{Results and discussion}

Data are available at osf.io/kgr2t. The data of primary interest are the number of words correctly recalled from each processing condition (see Table 4 and Fig. 6). In a planned analysis, a 3 (processing condition: date vs. housewarming vs. pleasantness) $\times 2$ (participant sex: male vs. female) between-groups ANOVA showed a main effect of processing condition, $F(2,106)=$ $17.52, p<.001, \eta_{\mathrm{p}}{ }^{2}=.25$. There was no significant main effect of participant sex, $F(1,106)=0.58, p=.45$, and no interaction between processing condition and sex, $F(2,106)=1.79, p=.17$. We used independent- samples $t$ tests for pairwise comparisons between the processing conditions. A comparison between the date condition and the pleasantness condition showed significantly higher recall of traits processed in the date condition, $t(73)=5.16, p<.001$, Cohen's $d=1.21$. There were also significantly more traits recalled in the housewarming condition than in the pleasantness condition, $t(72)=5.43, p<.001$, Cohen's $d=1.28$. There was no significant difference between the date and housewarming conditions, $t(73)=0.21, p=.83$. Intrusions were coded the same way as for Experiments 1 and 2, and a one-way ANOVA showed no significant difference in the intrusion rate across processing conditions, $F(2,109)=0.20, p=.82$.

Turning to the secondary data of interest, we conducted planned comparisons on the average ratings and response times from each processing condition (see Table 4). A one-way between-groups ANOVA on the ratings showed a significant difference, $F(2,109)=$ 41.34, $p<.001, \eta_{\mathrm{p}}{ }^{2}=.43$. In pairwise comparisons, ratings in the housewarming condition were significantly higher than those in the date condition, $t(73)=2.76$, $p<.01$, Cohen's $d=0.65$. Ratings in the pleasantness condition were significantly higher than in the date condition, $t(73)=9.18, p<.001$, Cohen's $d=2.15$, as well as the housewarming condition, $t(72)=6.07, p<.001$, Cohen's $d=1.43$. The same type of ANOVA on the response times also showed a significant difference between conditions, $F(2,109)=8.36, p<.001, \mathrm{n}_{\mathrm{p}}{ }^{2}=.13$, with response times being faster in the pleasantness condition than in the date condition, $t(73)=3.40, p<.001$, Cohen's $d=$ 0.80 , as well as in the housewarming condition, $t(72)=4.05$, $p<.001$, Cohen's $d=0.95$. (Although the effect sizes are large, the actual average differences are less than half a second.) There was no significant difference in response times between the date and housewarming conditions, $t(73)=0.17$, $p=.87$.

In an unplanned items analysis, we explored the relationship between relevance ratings and recall by calculating Pearson correlations between average ratings and recall for each stimulus in each processing condition. Unlike in previous experiments, relevance ratings were significantly related to recall in the date condition,

Table 4 Descriptive statistics for Experiment 3

\begin{tabular}{|c|c|c|c|c|c|c|c|c|c|}
\hline \multirow[b]{2}{*}{ Processing condition } & \multirow[b]{2}{*}{$n$} & \multicolumn{2}{|l|}{ Recall } & \multicolumn{2}{|c|}{ Intrusions } & \multicolumn{2}{|c|}{ Ratings } & \multicolumn{2}{|c|}{ Response times (ms) } \\
\hline & & $M$ & $S D$ & $M$ & $S D$ & $M$ & $S D$ & $M$ & $S D$ \\
\hline Date & 38 & 15.13 & 3.98 & 2.08 & 1.26 & 2.38 & 0.46 & $1,880.59$ & 388.47 \\
\hline Housewarming & 37 & 15.32 & 3.89 & 2.35 & 1.98 & 2.67 & 0.47 & $1,894.31$ & 316.93 \\
\hline Pleasantness & 37 & 10.73 & 3.37 & 2.16 & 2.33 & 3.30 & 0.42 & $1,617.07$ & 270.04 \\
\hline
\end{tabular}




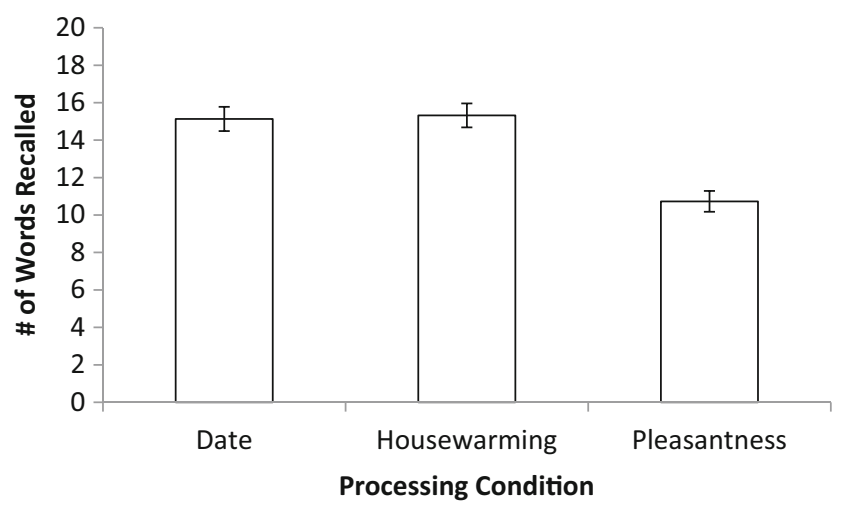

Fig. 6 Average number of words recalled from each processing condition in Experiment 3. Error bars depict standard error of the mean. The $y$-axis is truncated for clarity; the maximum possible number of words that could be recalled is 32

$r(31)=.57, p=.001$, and the housewarming condition, $r(31)=.36, p=.04$, but not the pleasantness condition, $r(29)=-.23, p=.49$. There were fewer significant correlations between ratings of stimuli in other conditions compared to previous experiments; only the correlation between ratings in the date and pleasantness conditions was significant, $r(31)=.57, p=.001$. Correlations between the date and housewarming conditions, $r(31)=.19, p=.31$, and the housewarming and pleasantness conditions, $r(31)=.11, p=.56$, were not significant.

As in Experiment 1, we examined whether the relevance ratings could be influencing the recall pattern through an ANCOVA with ratings as a covariate. Average ratings in this experiment did not significantly affect recall, $F(1,108)=1.06, p=.31$. The main effect of processing condition remained significant, $F(2,108)=14.10, p<.001, \eta_{\mathrm{p}}^{2}=.21$. Again, this suggests the recall results are likely not being driven by the relevance ratings.

The results of Experiment 3 do not seem to provide evidence of a reproductive advantage in memory. Processing words in the context of gifts on a romantic date (which likely has some relevance to reproduction) did not improve memory compared to processing words in the context of a housewarming party (likely less relevant to reproduction), although both processing conditions did improve recall compared to a traditional pleasantness rating task. One could make an argument that a scenario involving giving a housewarming gift is still somewhat relevant to evolutionary fitness, in that giftgiving could take place in the context of social exchange, reciprocal altruism, displays of wealth, or other mainstays of evolutionary theory. However, it is probably more likely that a scenario involving gift-giving on a romantic date more directly relates to evolutionary fitness. A shortcoming in the experiment is that both the romantic date scenario and the housewarming scenario produced significant correlations between relevance ratings and recall, but the housewarming scenario produced higher relevance ratings than the romantic date scenario. Both of those considerations could have reduced the probability of detecting a recall difference between the conditions if one existed. The lack of difference in recall between these two conditions could also be explained by claiming that both the romantic date scenario and the housewarming scenario involve a rich, distinctive type of processing without necessarily invoking any kind of fitness-relevant processing explanation (see General Discussion).

\section{General discussion}

The goal of the present study was to examine whether processing stimuli for their reproductive value would produce a recall benefit similar to processing stimuli for their survival value (Nairne \& Pandeirada, 2008b). Previous research has suggested that memory systems do show evidence of sensitivity to information relevant to reproduction (Kole \& Healy, 2011; Smith et al., 2013; Smith et al., 2012), but Sandry et al. (2013) found no evidence that various types of fitnessrelevant processing (including reproduction) benefitted memory to the same degree as survival processing. We speculated that one potential reason that Sandry et al. failed to find evidence of a reproductive processing advantage in memory was that their processing scenarios dealt with rating objects for their relevance in obtaining sexual partners, and objects may be much less relevant to reproduction than survival in the way the scenarios were presented to participants. In Experiments 1 and 2, we asked participants to rate traits of potential sexual partners with the goal that this task would be more relevant to real-life reproduction scenarios. (As we discuss below, we did not find strong evidence that rating adjectives is indeed more relevant than rating objects in reproduction scenarios.) We also utilized an object-rating task in Experiment 3 in an attempt to more realistically address the role that objects could play in courtship (as gifts).

In Experiment 1, participants rated traits for their relevance in a long-term mate, a coworker, or how pleasant each word was. Results showed a trend toward recalling more traits in the mate processing condition, in that mate processing produced better recall than pleasantness processing, but coworker processing did not differ significantly from mate processing or pleasantness 
processing. Regarding our assumption that rating adjectives of potential mates is more relevant than Sandry et al.'s (2013) task involving rating objects for their relevance in mate selection, we did not find large differences between our relevance ratings and Sandry et al.'s as one might have expected if our assumption were true. Mean ratings of adjectives in our mate processing condition of Experiment 1 were comparable to the mate selection conditions of Sandry et al.'s experiments. These comparisons come with important caveats, such as the various other differences between stimulus sets, but we did not obtain the reproductive processing advantage in recall performance that we might have if stimulus relevance were an issue

We also thought it was possible that Experiment 1 may have failed to activate the strongest aspects of reproductive processing, one of which has been wellestablished to differ by sex: jealousy toward infidelity (Buss et al., 1992). In Experiment 2, we combined the adaptive memory paradigm with the phenomenon of sex differences in jealousy in order to explore adaptive memory using a well-replicated finding in evolutionary psychology. Participants rated traits for their relevance in detecting sexual infidelity, emotional infidelity, or how pleasant each word was. We replicated the basic effect of sex differences in jealousy, in that men were more likely than women to report that sexual infidelity would be more distressing, but the results showed no differences in recall across processing conditions. There were several unpredicted interactions between the sex of the participant and the response to the jealousy question (Experiment 2a) and between the sex of the participant, the processing condition and the response to the jealousy question, as well as a main effect of women recalling more traits overall than men (Experiment 2b). These results suggest that memory may be sensitive to certain conditions depending on the scenario and characteristics of the participant, but the analyses involved small numbers of participants in some conditions, and the results are difficult to explain under a coherent theoretical framework. Following up on the issue of whether rating adjectives was more relevant than rating objects for reproductive processing, ratings in our jealousy conditions of Experiment 2 were comparable to similar conditions (such as mate selection, jealousy, and infidelity) in Sandry et al.'s (2013) Experiment 1a, which used a similar within-subjects design. Recall the current Experiment 2 (2.46-4.60 out of 20) was comparable to Sandry et al.'s Experiment 1a (1.33-2.39 out of 10), which was still somewhat low. Again, there were various other differences in stimulus sets and design between the two sets of experiment, but we interpret the current results as a lack of strong support that processing adjectives is more relevant than rating objects in reproductive processing.

Finally, in Experiment 3 we constructed scenarios in which objects could be relevant to reproduction. Participants rated the appropriateness of gifts in the context of a romantic date or a housewarming party. Both date processing and housewarming processing improved recall compared to pleasantness ratings but recall did not differ significantly between date and housewarming processing. Despite our intention of using nouns in a processing condition that would be relevant to reproductive processing, the ratings in Experiment 3 were not higher than the ratings of adjectives in Experiments 1 and 2.

There are several possible explanations for the current findings. Given that in evolutionary theory survival is only important inasmuch as it allows for more mating opportunities or enhancing inclusive fitness, it seems unlikely that memory systems would be sensitive to factors important for survival but not reproduction. One possibility is that memory does benefit from reproductive processing, but the effect was masked in the current experiments by comparison tasks which were themselves evolutionarily relevant. For example, in Experiment 1, mate processing produced better recall than pleasantness processing but not coworker processing. The goal was to compare a reproductively relevant scenario (evaluating potential mates) and a nonreproductively relevant scenario (evaluating potential colleagues). But evaluating the characteristics of longtime collaborators and professional partners may have been incidentally evolutionarily relevant to the degree that the characteristics of one's colleagues may affect one's own long-term professional successes and therefore one's standing in social hierarchies and access to mating opportunities. Selection of nonmate social companions with whom to form coalitions could conceivably be vital for survival in an ancestral environment (Bugental, 2000). Support for the possibility that coworker processing might have had some evolutionary relevance is that in Experiment 1 the relevance ratings for stimuli in each condition correlated very highly with the relevance ratings in other conditions, showing that traits relevant to mate selection are also relevant to coworker relevance (and that both are perceived as pleasant traits). This unintentional evolutionary relevance could have accounted for the intermediate memory performance between mate processing and pleasantness processing.

In Experiment 3, there was again no difference between recall between the condition we intended to be evolutionarily relevant (date processing) and the condition we intended not to be evolutionarily relevant (housewarming processing). One explanation could be that choosing a gift involves planning and foresight, 
and therefore recruits a set of adaptive cognitive mechanisms (e.g., Klein, Robertson, \& Delton, 2011). Further, it has been argued that reasoning about object function is a potent proximate mechanism for effective encoding of stimuli. Bell, Röer, and Buchner (2015) found that emphasis on object function in Nairne et al.'s (2007) original memory task led to better recall than emphasis on survival threat, underscoring the mnemonic value of this kind of processing. Evaluating the appropriateness of a gift for both scenarios may elicit object function processing, which could explain the lack of recall differences in Experiment 3. It could also be argued that both gift-giving conditions involve processing for social goals, which is ultimately in the interests of reproductive fitness. However, unlike in Experiment 1's correlation in ratings between the mate condition and the coworker condition, the ratings in the date condition were not significantly correlated to ratings in the housewarming condition. This supports the possibility that the criteria are indeed different for gifts on romantic dates and housewarming parties, even if they did not produce differences in recall.

Another potential concern surrounding the relevance ratings is whether differences in ratings could have diminished or eliminated recall differences. In Experiment 1, recall was numerically higher in the mate condition than in the coworker and pleasantness conditions, but the ratings were lower. In Experiment 2, the jealousy conditions produced higher ratings than pleasantness processing (most likely due to the possibility that traits that are relevant to detecting infidelity are unpleasant), but recall did not differ across conditions. In Experiment 3, ratings were lower in the date condition than in the housewarming or pleasantness conditions, despite no difference in recall between the date and housewarming conditions. It is somewhat concerning to see such variability in ratings differences between conditions that are supposed to be comparable, but these effects are likely not masking a reproductive processing effect. Analyses in all experiments failed to find any significant effect of recall when relevance ratings were controlled for, and although prior work (Butler et al., 2009) has shown that scenario-congruent materials are recalled better than scenario-incongruent materials, it is possible for a condition to produce lower ratings than comparison conditions but higher recall (e.g., Nairne et al., 2007, Experiment. 1 and 4; Kang et al., 2008, Experiments 1 and 3).

In Experiment 2, one obvious impediment to detecting a reproductive processing effect is the low recall rates, similar to what Sandry et al. (2013) obtained. This floor effect could simply be due to the lowfrequency stimuli. Recall increased slightly between Experiment $2 \mathrm{a}$ and Experiment $2 \mathrm{~b}$, in which the stimuli had a higher word frequency. However, the still-low recall rates could also be attributable to the abstractness of the stimuli. It has long been established that concrete (easy to visualize) words are recalled more easily than abstract (difficult to visualize) words (Paivio, 1965). Because adjectives are more abstract than nouns, detecting a reproductive processing advantage in memory may not be as straightforward as substituting nouns in a survival scenario with adjectives in a mate-selection scenario. Support from this possibility comes from previous findings in which using pictures as stimuli in a survival processing task produced higher recall compared to a moving scenario, as well as overall higher recall compared to when the stimuli were words (Otgaar et al., 2010). It has also been shown that the survival advantage appears for concrete, but not abstract words, even though abstract words receive higher relevance ratings than concrete words (Bell, Röer, \& Buchner, 2013).

An important consideration in the current results is the high number of intrusions, especially in Experiments 1 and 2, compared to previous research (e.g., Butler et al., 2009; Kroneisen, Erdfelder, \& Buchner, 2013; Nairne et al., 2007). Nairne et al. suggested that if participants generate recall attempts based on category membership or schemata one might expect a higher intrusion rate, and that may have occurred in the current experiments. In other words, participants may have been generating random characteristics that could describe people as opposed to recalling stimuli from earlier in the experiment. If participants were indeed generating characteristics instead of recalling them then the encoding manipulations in the current experiments were likely not as influential as we had intended. Support for this possibility is the lack of a congruity effect in Experiments 1 and 2, in which items that received higher relevance ratings were not necessarily recalled better. Interestingly, Experiment 3 did produce a congruency effect, and the number of intrusions declined. Overall, these considerations may make the current data difficult to interpret.

One more possibility is that human memory is sensitive to reproductively-relevant information but that traits about individuals are less informative about sexual behavior than other things. For example, considering whether an individual is artistic may or may not be helpful in evaluating that person's reproductive potential. However, considering a person's behaviors involving past sexual partners or current interactions with reproductive rivals may be the kind of fitnessrelevant information to which memory systems are more tuned.

One final possible explanation for the current findings is that a reproductive processing effect in memory 
did not appear because it is a theoretical extension of the survival processing effect, and the survival processing itself may not necessarily reflect natural selection's influence on memory systems. To clarify, this is not suggesting that evolution "stops at the neck" or that memory systems do not contain evolutionary adaptations. Rather, it is possible that the survival processing effect can be explained through general memory principles, which are themselves the adaptations, and that it is not essential for processing conditions to invoke fitnessrelevance (Howe \& Otgaar, 2013), or that survival processing is an exaptation (co-opting) of basic memory processes (Nairne \& Pandeirada, 2016). Several studies have recently explored the proximate mechanisms behind why survival processing produces superior memory performance. For example, Kroneisen et al. (2013) argued that survival processing improves memory simply because it involves richness and distinctiveness in encoding and interactive imagery, which themselves have been shown to improve memory. Kroneisen and Erdfelder (2011) showed that when survival scenarios are reduced to involve only one problem (e.g., finding water) instead of three (finding water, food, and avoiding predators) the memory advantage diminishes. Kroneisen and Erdfelder claimed that reducing scenarios should affect the richness of the scenario but not the fitness relevance of the processing, so the memory advantage is due to something other than fitness relevance. Röer, Bell, and Buchner (2013) showed that the survival processing effect can be explained by invoking the number of ideas generated for each item in various scenarios and the congruence of those items with the scenario, which also supports the richness of encoding hypothesis. Burns, Hwang, and Burns (2011) also examined the role of item-specific and relational processing in the survival task, but they suggest that it is possible that these mechanisms themselves are the adaptations that promote memory for survival situations. However, McBride, Thomas, and Zimmerman (2013) argued that if survival processing serves adaptive needs it might have evolved in an evolutionarily older form of memory, but they found no evidence of a survival processing advantage in implicit memory. Some research has even shown that processing in dying scenarios can benefit memory to a comparable degree as survival processing (Burns, Hart, \& Kramer, 2014), which may call fitness-relevancy into question. But as Nairne and Pandeirada (2016) point out, even though findings such as these might suggest survival processing is an exaptation of other processes, adaptations often interact with other processes to reach adaptive goals, so identifying core processes in a phenomenon is not the only important part of explaining the phenomenon. Furthermore, ultimate and proximate explanations of a phenomenon are complementary (Scott-Phillips, Dickins, \& West, 2011), so identifying the proximate mechanisms of the survival processing effect does not necessarily detract from the ultimate function.

The current study failed to find strong evidence that fitness-relevant processing benefits memory. However, there is a growing body of research that has successfully expanded the functionalist view of memory to concepts such as animacy (Bonin, Gelin, \& Bugaiska, 2014; Nairne, VanArsdall, Pandeirada, Cogdill, \& LeBreton, 2013; VanArsdall, Nairne, Pandeirada, \& Blunt, 2013) and risk of contamination from disease (Nairne, 2015). The current results are also in contrast to other studies that have specifically demonstrated sensitivity in memory to information relevant to mating (Kole \& Healy, 2011; Smith et al., 2013; Smith et al., 2012). It is possible that processing verbal materials (which received low relevance ratings in many of the current experiments) may not be the best way to evaluate fitness relevance. More work is necessary to examine what other types of reproductive processing can benefit memory, and whether they utilize the same proximate mechanisms as the survival processing advantage. For example, Klein (2013) found that processing in survival scenario produced better recall than a mate selection scenario, and argued the reason was because survival processing is more oriented toward planning than mate selection (Klein, Robertson, \& Delton, 2010, 2011). An interesting extension of these findings could involve mate selection tasks that do somehow invoke future-oriented planning.

Other possible avenues could be processing pictures in terms of reproductive relevance. These types of manipulations have sometimes produced survival processing advantages (Otgaar et al., 2010) and sometimes not (Savine, Scullin, \& Roediger, 2011). But with imagery being an important part of mate selection (at least for men), it is possible that memory systems could be sensitive to visual information pertaining to who would be good potential mates. Another possibility is to examine behaviors as in the context of mate selection, which could reveal that memory systems are finetuned for behavioral indicators of reproductive potential (as opposed to personality characteristics or material resources).

Acknowledgements This research was supported by the First Summer Research Support from the College of Health and Human Services at Missouri State University, awarded to Bogdan Kostic. Special thanks to Dalton Anderson, Taylor Barthels, Leslie Bland, Brooke Clodfelter, Elizabeth M. Delong, Ashley Torkelson, and Ryan Trickey for assisting in data collection. 
Appendix A

Table 5 Stimuli for each experiment

\begin{tabular}{|c|c|c|}
\hline \multicolumn{3}{|l|}{ Experiment 1} \\
\hline antisocial & funky & mediocre \\
\hline apologetic & gifted & moody \\
\hline arty & grim & polite \\
\hline carefree & grumpy & reliable \\
\hline cautious & gutsy & restless \\
\hline childish & healthy & sheepish \\
\hline classy & humble & silent \\
\hline compassionate & idiotic & skilled \\
\hline eager & insensitive & sociable \\
\hline forgetful & lazy & stylish \\
\hline \multicolumn{3}{|l|}{ Experiment $2 \mathrm{a}$} \\
\hline able & homely & slothful \\
\hline ashy & icy & spiritless \\
\hline attentive & illiterate & stagnant \\
\hline bigoted & incredible & stouthearted \\
\hline brainy & instinctive & subversive \\
\hline broad & invaluable & suggestive \\
\hline cautious & limitless & superb \\
\hline chatty & lonely & supple \\
\hline cocky & malicious & surreal \\
\hline corruptible & meek & temperamental \\
\hline deceitful & merry & unadjusted \\
\hline dejected & mischievous & unchangeable \\
\hline disorderly & muted & undaunted \\
\hline dispassionate & paranoid & unemployable \\
\hline downhearted & pestilent & ungratified \\
\hline expressible & playful & unorthodox \\
\hline flawless & primitive & unsullied \\
\hline graceless & pudgy & untiring \\
\hline handy & puny & upbeat \\
\hline hapless & simple & wishful \\
\hline \multicolumn{3}{|l|}{ Experiment $2 b$} \\
\hline adventurous & dependable & noble \\
\hline aggressive & depressed & nosey \\
\hline annoying & dramatic & obedient \\
\hline anxious & dull & optimistic \\
\hline arrogant & emotional & picky \\
\hline artistic & entertaining & polite \\
\hline awkward & fearless & poor \\
\hline boring & funny & quirky \\
\hline bossy & grateful & reliable \\
\hline calm & handy & rude \\
\hline caring & helpful & sad \\
\hline cheerful & humble & sarcastic \\
\hline clever & immature & selfish \\
\hline conceited & independent & smart \\
\hline
\end{tabular}

Table 5 (continued)

\begin{tabular}{llll}
\hline $\begin{array}{l}\text { confident } \\
\text { controlling }\end{array}$ & lazy & sneaky & \\
cool & loud & spiritual & \\
creative & messy & stubborn & \\
cruel & modest & talkative & \\
$\begin{array}{c}\text { curious } \\
\text { Experiment } 3\end{array}$ & needy & witty & \\
ashtray & coffee & magazines & plant \\
bicycle & corkscrew & meat & poem \\
blender & cosmetics & music & popcorn \\
book & dishes & pajamas & pottery \\
candle & doll & pencils & soap \\
cash & flowers & perfume & socks \\
cheese & jewelry & pet & sweater \\
clock & keychain & picture & wine \\
\hline
\end{tabular}

\section{Appendix B: Instructions for each condition in Experiment 2}

\section{Sexual jealousy condition}

For this task, please think of a serious committed romantic relationship that you have had in the past, that you currently have, or that you would like to have.

Imagine that you suspect this partner-with whom you've been seriously involved-is having passionate sexual intercourse with someone else.

We are going to show you a list of traits that your partner might have, and we would like you to rate how helpful each of these words would be for you in finding out if your partner is having passionate sex with someone else. Some of these words may be helpful, others may not-it's up to you to decide.

Each word will appear for only 10 seconds, so make sure you give your rating in that time.

Press the SPACE bar for an example.

\section{Emotional jealousy condition}

For this task, please think of a serious committed romantic relationship that you have had in the past, that you currently have, or that you would like to have.

Imagine that you suspect this partner-with whom you've been seriously involved - is falling in love with someone else.

We are going to show you a list of traits that your partner might have, and we would like you to rate how helpful each of these words would be for you in finding out if your partner is falling in love with someone else. Some of these words may be helpful, others may not-it's up to you to decide.

Each word will appear for only 10 seconds, so make sure you give your rating in that time.

Press the SPACE bar for an example. 


\section{Pleasantness rating condition}

For this task, we are going to show you a list of words, and we would like you to rate the pleasantness of each word. Some of these words may be pleasant, others may not - it's up to you to decide.

Each word will appear for only 10 seconds, so make sure you give your rating in that time.

Press the SPACE bar for an example.

\section{References}

Bailey, J. M., Gaulin, S., Agyei, Y., \& Gladue, B. A. (1994). Effects of gender and sexual orientation on evolutionarily relevant aspects of human mating psychology. Journal of Personality and Social Psychology, 66, 1081-1093. doi:10.1037/0022-3514.66.6.1081

Balota, D. A., Yap, M. J., Cortese, M. J., Hutchison, K. A., Kessler, B., Loftis, B., ... \& Treiman, R. (2007). The English lexicon project. Behavior Research Methods, 39, 445-459. doi:10.3758/ BF03193014

Bell, R., Röer, J. P., \& Buchner, A. (2013). Adaptive memory: The survival-processing memory advantage is not due to negativity or mortality salience. Memory \& Cognition, 41, 490-502. doi:10.3758/ s13421-012-0290-5

Bell, R., Röer, J. P., \& Buchner, A. (2015). Adaptive memory: Thinking about function. Journal of Experimental Psychology: Learning, Memory, \& Cognition, 41, 1038-1048. doi:10.1037/xlm0000066

Bonin, P., Gelin, M., \& Bugaiska, A. (2014). Animates are better remembered than inanimates: Further evidence from word and picture stimuli. Memory \& Cognition, 42, 370-382. doi:10.3758/s13421-013-0368-8

Bugental, D. B. (2000). Acquisition of the algorithms of social life: A domain-based approach. Psychological Bulletin, 126, 187 219. doi:10.1037//0033-2909.126.2.187

Burns, D. J., Hart, J., \& Kramer, M. E. (2014). Dying scenarios improve recall as much as survival scenarios. Memory, 22(1), 51-64. doi:10. 1080/09658211.2013.795973

Burns, D. J., Hwang, A. J., \& Burns, S. A. (2011). Adaptive memory: Determining the proximate mechanisms responsible for the memorial advantages of survival processing. Journal of Experimental Psychology: Learning, Memory, \& Cognition, 37(1), 206-2018. doi: $10.1037 / \mathrm{a} 0021325$

Buss, D. M., Larsen, R. J., Westen, D., \& Semmelroth, J. (1992). Sex differences in jealousy: Evolution, physiology, and psychology. Psychological Science, 3(4), 251-255. doi:10.1111/j.1467-9280. 1992.tb00038.x

Butler, A. C., Kang, S. H. K., \& Roediger, H. L. (2009). Congruity effects between materials and processing tasks in the survival processing paradigm. Journal of Experimental Psychology: Learning, Memory, \& Cognition, 35(6), 1477-1486. doi:10.1037/a0017024

Craik, F. I. M., \& Tulving, E. (1975). Depth of processing and the retention of words in episodic memory. Journal of Experimental Psychology: General, 104(3), 268-294. doi:10.1037/0096-3445.104.3.268

Hall, J. F. (1954). Learning as a function of word frequency. The American Journal of Psychology, 67, 138-140. doi:10.2307/1418080

Harris, C. R. (2003). A review of sex differences in sexual jealousy, including self-report data, psychophysiological responses, interpersonal violence, and morbid jealousy. Personality and Social Psychology Review, 7(2), 102-128. doi:10.1207/S15327957PSPR0702 102-128

Howe, M. L., \& Otgaar, H. (2013). Proximate mechanisms and the development of adaptive memory. Current Directions in Psychological Science, 22(1), 16-22. doi:10.1177/0963721412469397
Kang, S. H., McDermott, K. B., \& Cohen, S. M. (2008). The mnemonic advantage of processing fitness-relevant information. Memory \& Cognition, 36(6), 1151-1156. doi:10.3758/MC.36.6.1151

Klein, S. B. (2013). Does optimal recall performance in the adaptive memory paradigm require the encoding context to encourage thoughts about the environment of evolutionary adaptation? Memory \& Cognition, 41, 49-59. doi:10.3758/s13421-012-0239-8

Klein, S. B., Robertson, T. E., \& Delton, A. W. (2010). Facing the future: Memory as an evolved system for planning future acts. Memory \& Cognition, 38(1), 13-22. doi:10.3758/MC.38.1.13

Klein, S. B., Robertson, T. E., \& Delton, A. W. (2011). The futureorientation of memory: Planning as a key component mediating the high levels of recall found with survival processing. Memory, 19(2), 121-139. doi:10.1080/09658211.2010.537827

Kole, J. A., \& Healy, A. F. (2011). Memory for details about people: Familiarity, relatedness, and gender congruency. Memory \& Cognition, 39(4), 637-648. doi:10.3758/s13421-010-0051-2

Kostic, B., McFarlan, C. C., \& Cleary, A. M. (2012). Extensions of the survival advantage in memory: Examining the role of ancestral context and implied social isolation. Journal of Experimental Psychology: Learning, Memory, \& Cognition, 38, 1091-1098. doi: 10.1037/a0026974

Kroneisen, M., \& Erdfelder, E. (2011). On the plasticity of the survival processing effect. Journal of Experimental Psychology: Learning, Memory, \& Cognition, 37(6), 1553-1562. doi:10.1037/a0024493

Kroneisen, M., Erdfelder, E., \& Buchner, A. (2013). The proximate memory mechanism underlying the survival-processing effect: Richness of encoding or interactive imagery? Memory, 21(4), 494-502. doi: 10.1080/09658211.2012.741603

McBride, D. M., Thomas, B. J., \& Zimmerman, C. (2013). A test of the survival processing advantage in implicit and explicit memory tests. Memory \& Cognition, 41, 862-871. doi:10.3758/s13421-013-0304-y

Miller, G. F. (2000). The mating mind: How sexual choice shaped the evolution of human nature. New York: Doubleday.

Nairne, J. S. (2015). Adaptive memory: Novel findings acquired through forward engineering. In D. S. Lindsay, C. M. Kelley, A. P. Yonelinas, \& H. L. Roediger (Eds.), Remembering: Attributions, processes, and control in human memory (pp. 3-14). New York: Psychology Press.

Nairne, J. S., \& Pandeirada, J. N. S. (2008a). Adaptive memory: Is survival processing special? Journal of Memory \& Language, 59(3), 377-385. doi:10.1016/j.jml.2008.06.001

Nairne, J. S., \& Pandeirada, J. N. S. (2008b). Adaptive memory: Remembering with a stone-age brain. Current Directions in Psychological Science, 17(4), 239-243. doi:10.1111/j.1467-8721. 2008.00582.x

Nairne, J. S., \& Pandeirada, J. N. S. (2010). Adaptive memory: Ancestral priorities and the mnemonic value of survival processing. Cognitive Psychology, 61, 1-22. doi:10.1016/j.cogpsych.2010.01.005

Nairne, J. S., \& Pandeirada, J. N. S. (2016). Adaptive memory: The evolutionary significance of survival processing. Perspectives on Psychological Science, 11(4), 496-511. doi:10.1177/ 1745691616635613

Nairne, J. S., Pandeirada, J. N. S., Gregory, K. J., \& VanArsdall, J. E. (2009). Adaptive memory: Fitness-relevance and the huntergatherer mind. Psychological Science, 20(6), 740-746. doi:10. 1111/j.1467-9280.2009.02356.x

Nairne, J. S., Pandeirada, J. N. S., \& Thompson, S. R. (2008). Adaptive memory: The comparative value of survival processing. Psychological Science, 19(2), 176-180. doi:10.1111/j.1467-9280. 2008.02064.x

Nairne, J. S., Thompson, S. R., \& Pandeirada, J. N. (2007). Adaptive memory: Survival processing enhances retention. Journal of Experimental Psychology: Learning, Memory, \& Cognition, 33(2), 263-273. doi:10.1037/0278-7393.33.2.263 
Nairne, J. S., VanArsdall, J. E., Pandeirada, J. N. S., Cogdill, M., \& LeBreton, J. M. (2013). Adaptive memory: The mnemonic value of animacy. Psychological Science, 24, 2099-2105. doi:10.1177/ 0956797613480803

Otgaar, H., Smeets, T., \& van Bergen, S. (2010). Picturing survival memories: Enhances memory after fitness-relevant processing occurs for verbal and visual stimuli. Memory \& Cognition, 38(1), 23-28. doi: 10.3758/MC.38.1.23

Paivio, A. (1965). Abstractness, imagery, and meaningfulness in pairedassociate learning. Journal of Verbal Learning \& Verbal Behavior, 4(1), 32-38. doi:10.1016/S0022-5371(65)80064-0

Röer, J. P., Bell, R., \& Buchner, A. (2013). Is the survival-processing memory advantage due to richness of encoding? Journal of Experimental Psychology: Learning, Memory, and Cognition, 39(4), 1294-1302. doi:10.1037/a0031214

Sagarin, B. J., Martin, A. L., Coutinho, S. A., Edlund, J. E., Patel, L., Skowronski, J. J., \& Zengel, B. (2012). Sex differences in jealousy: A meta-analytic examination. Evolution and Human Behavior, 33(6), 595-614. doi:10.1016/j.evolhumbehav.2012.02.006

Sandry, J., Trafimow, D., Marks, M. J., \& Rice, S. (2013). Adaptive memory: Evaluating alternative forms of fitness-relevant processing in the survival processing paradigm. PLOS ONE, 8(4), e60868. doi: 10.1371/journal.pone.0060868
Savine, A. C., Scullin, M. K., \& Roediger, H. L. (2011). Survival processing of faces. Memory \& Cognition, 39, 1359-1373. doi:10. 3758/s13421-011-0121-0

Schützwohl, A., \& Koch, S. (2004). Sex differences in jealousy: The recall of cues to sexual and emotional infidelity in personally more and less threatening context conditions. Evolution and Human Behavior, 25(4), 249-257. doi:10.1016/j.evolhumbehav2004.03.006

Scott-Phillips, T. C., Dickins, T. E., \& West, S. A. (2011). Evolutionary theory and the ultimate-proximate distinction in the human behavioral sciences. Perspectives on Psychological Science, 6, 38-47. doi: $10.1177 / 1745691610393528$

Smith, D. S., Jones, B. C., Feinberg, D. R., \& Allan, K. (2012). A modulatory effect of male voice pitch on long-term memory in women: Evidence of adaptation for mate choice? Memory \& Cognition, 40(1), 135-144. doi:10.3758/s13421-011-0136-6

Smith, D. S., Jones, B. C., \& Allan, K. (2013). Socio-sexuality and episodic memory function in women: Further evidence of an adaptive "mating mode.". Memory \& Cognition, 41(6), 850-861. doi:10. 3758/s13421-013-0301-1

VanArsdall, J. E., Nairne, J. S., Pandeirada, J. N. S., \& Blunt, J. R. (2013). Adaptive memory: Animacy processing produces mnemonic advantages. Experimental Psychology, 60, 172-178. doi:10.1027/16183169/a000186 